\title{
Spatial-frequency specificity in visual masking
}

\author{
CHARLES W. WHITE and CHARLES M. LORBER \\ Duke University, Durham, North Carolina 27706
}

\begin{abstract}
Visual masking between nonoverlapping gratings depends on the similarity of their spatial frequencies. In a metacontrast experiment, bar gratings of 6 and 12 cycles/deg were masked by flanking gratings of various spatial frequencies. For three observers, masking was greatest when the masking frequency was approximately equal to the masked frequency. The results are interpreted in terms of a theory of reciprocal inhibition among frequency-specific neural units.
\end{abstract}

When two visual stimuli occur in rapid succession, one sometimes masks the appearance of the other. Metacontrast is masking among visual stimuli which do not overlap spatially. (See Lefton, 1973, for a recent review.) We propose that one of the parameters that determines such masking is the similarity of the spatial frequency spectra, the extent to which the stimuli share spatial frequencies.

There is considerable evidence from psychophysical (Campbell, 1974; Graham \& Nachmias, 1971; Lovegrove \& Over, 1972) and physiological sources (Campbell, Cooper, Robson, \& Sachs, 1969; Maffei \& Fiorentini, 1973; Pollen \& Taylor, 1974) suggesting that mammalian visual systems perform a spatial-frequency analysis of the retinal illuminance distribution (Campbell \& Robson, 1968).

The general approach implies that channels in the visual system respond to different bands of spatial frequency and that many frequency-specific visual phenomena depend on the stimulation of subpopulations of these units. Examples include frequency-specific McCollough aftereffects (Stromeyer, 1972), motion aftereffects (Mayhew \& Anstis, 1972), visual masking in one dimension (Weisstein \& Bisaha, 1972), and in two dimensions (Harmon \& Julesz, 1973), as well as a visual analog of the auditory critical band phenomenon (Stromeyer \& Julesz, 1972).

Two previous studies have implicated spatial frequencies in other types of masking, but not in metacontrast. Weisstein and Bisaha (1972) showed that prior adaptation to gratings or single bars could reduce the apparent contrast of gratings in other parts of the visual field. Also, Armstrong and Sekuler (Note 1) used square-wave gratings as masks and targets in both overlapping and nonoverlapping conditions. Using a two-alternative forced-choice detection task, they showed a large masking effect with overlapping gratings, but their metacontrast conditions did not produce appreciable masking,

This research was supported in part by a Biological Sciences Support grant to Duke University, N.I.H. Grant 5505RR07070-09. Address correspondence to Charles $W$. White, Department of Psychology, Duke University, Durham, North Carolina 27706. perhaps because the forced-choice technique enabled observers to detect stimuli that were only partially masked.

The present study uses a more sensitive measure to investigate masking between nonoverlapping squarewave gratings and shows that some aspects of metacontrast are frequency-specific.

\section{METHOD}

\section{Observers}

The observers were three male students with normal or corrected vision who were paid for their participation.

\section{Apparatus}

The stimuli were constructed from matte-black chart tape mounted on white poster board. The patterns were photographed on high-contrast copy film and mounted in $2 \times 2$ in. slide mounts for presentation in a three-channel Maxwellian view optical system. All stimuli consisted of vertical bars, as shown in Figure 1. The target stimuli were square-wave gratings subtending $1 \times 3 \mathrm{deg}$ of visual

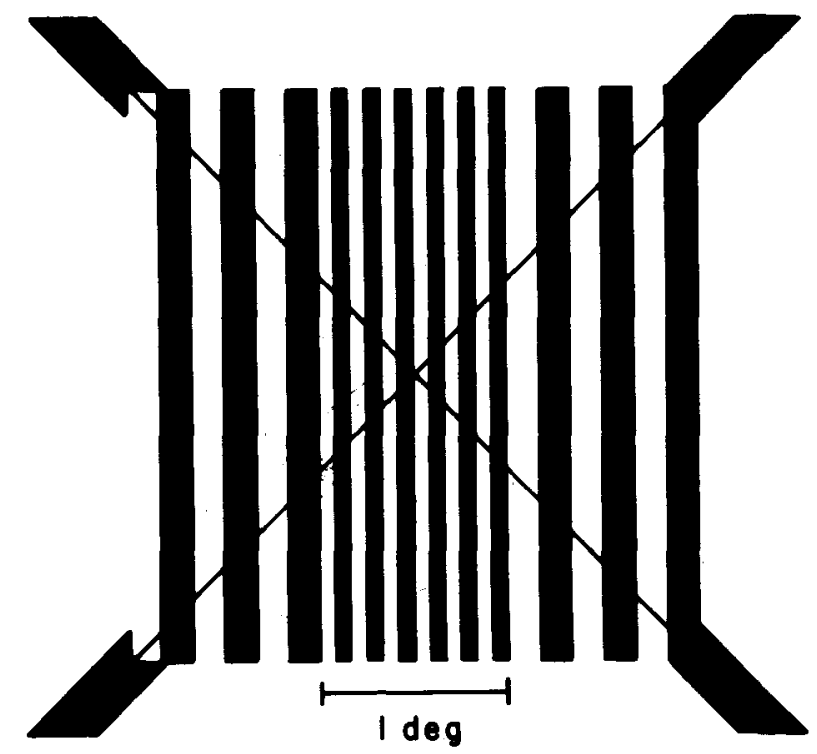

Figure 1. The stimulus field. The 6-cycle/deg target stimulus and the 3-cycle/deg masking stimulus would appear as shown above if they were simultaneously presented. The oblique lines are cross hairs for fixation. The entire display was centered within a 10-deg circular field. 


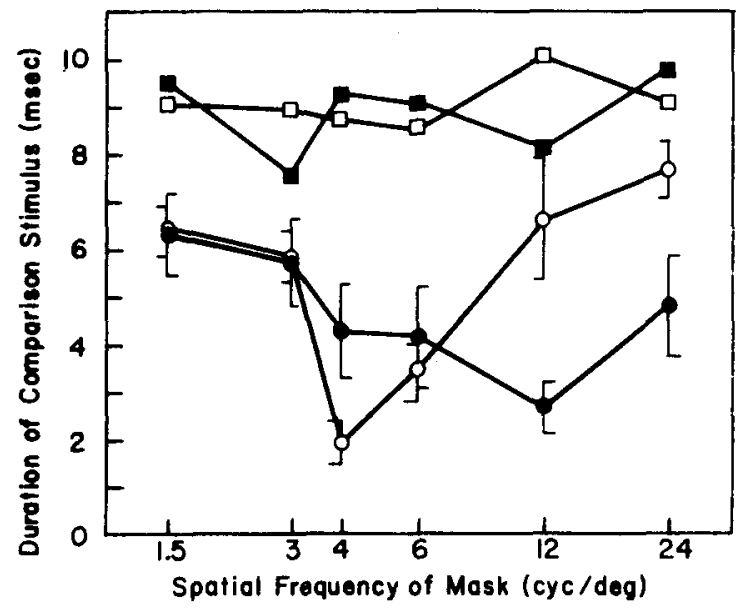

Figure 2. The ordinate indicates the duration of the comparison stimulus that matched the appearance of the target stimulus. Leas duration represents more masking. Vertical line segments mark 1 standard error above and below each mean. Circles indicate mean results for three observers with the masking stimuli presented, for the 6-cycle/deg target $(0)$ and for the 12 -cycle/deg target $(\bullet)$. Squares indicate results for two observers without the masking stimulus, for the 6 -cycle/deg target $(D)$ and the $12 \mathrm{cycle} / \mathrm{deg}$ target (a).

angle and containing either 6 or 12 cycles. Each masking stimulus had a total width of $3 \mathrm{deg}$. with a 1-deg gap in the center. Masks consisted of square-wave gratings of $1.5,3,4,6,12$, and 24 cycles $/ \mathrm{deg}$. The mean luminance of the target gratings was approximately $25 \mathrm{~cd} / \mathrm{m}^{2}$; masking gratings were approximately $11 \mathrm{~cd} / \mathrm{m}^{2}$. The contrast of each stimulus was 0.78 . Contrast was detined as (maximum luminance - minimum luminance)/ (maximum luminance + minimum luminance).

The gratings were superimposed on a fixation field of $0.05 \mathrm{~cd} / \mathrm{m}^{2}$. Dark cross hairs. less than 1 min of visual angle, were centered in the field, and thicker lines. $9.5 \mathrm{~min}$ wide, were in the periphery, as shown in Figure 1. The fixation field remained on during the entire session and assisted the observers both in fixating the targets and in maintaining the proper accommodation. The other two channels contained the target and mask stimuli. Illumination of each channel was provided by red light-emitting diodes (Monsanto, MV-50) with a wavelength distribution centered at $650 \mathrm{~nm}$ and a half-width of approximately $40 \mathrm{~nm}$. The light sources were selected for their rapid onset and offset characteristics.

\section{Procedure}

All stimuli were presented monocularly to the right eye. Each observer bit into a biteboard covered with dental impression compound and used a foreheadrest. The stimuli were presented in the following sequence: After 2 min of adapting to the fixation field. the observer initiated the run. The target stimulus (TS) appeared for $10 \mathrm{msec}$. After a delay of $90 \mathrm{msec}$, the masking stimulus (MS) was presented for $20 \mathrm{msec}$. The onset asynchrony between the two stimuli was chosen in a preliminary experiment in which an onset asynchrony of $100 \mathrm{msec}$ produced a high degree of masking with gratings. Approximately $1 \mathrm{sec}$ later, a comparison stimulus (CS) appeared for some duration between 1 and $15 \mathrm{msec}$. The $C S$ was the same grating as the TS. The observer was instructed to press one of two keys to indicate which pattern appeared clearer and more distinct: TS or CS. The observer's decision was based on such factors as the apparent contrast between the light and dark bars of the gratings. the sharpness of the contours, and whether any bars or pieces of bars appeared to be missing from the TS. Two observers also ran control trials, which were the same except that the MS was omitted.
The degree of masking was measured by a staircase psychophysical procedure. The durations of the TS and MS remained constant. but the CS duration was varied, depending on the observer's previous responses, so as to reach a point of subjective equality with the TS. As the CS duration was made shorter. the grating appeared dimmer and less clear than at longer durations. Thus the CS duration at the end of the staircase was a measure of the masking effect: the shorter the CS, the greater the masking.

Each series consisted of six presentations: two control trials. two masking trials from a staircase in which the initial CS duration was 15 msec. and two masking trials from a staircase which started at 5 msec. The control trials were added after one observer completed the experiment. The CS duration was changed only if both responses to a stimulus in a series were the same. For example, two responses that the CS appeared less clear than the TS caused the duration of the $\mathrm{CS}$ to be increased by $1 \mathrm{msec}$ in the next series. If the two responses to the same stimulus in a series were not identical. then the CS duration was unchanged for the next series. Each session, consisting of 30 series, required about $20 \mathrm{~min}$ to complete. The first 15 served as practice and brought the staircases within the proper range; the CS durations during the second 15 series were averaged, and data from both staircases in a given session were pooled. Randomization and presentation of stimuli, as well as the determination of CS durations. were controlled by a computer. Each observer completed six practice sessions before the data were collected.

\section{RESULTS}

The case for spatial-frequency specificity in metacontrast is summarized in Figure 2. The upper pair of curves plots the mean results of the control trials, during which no masking stimulus was presented, for two of the observers. Since one observer did not run the control series, it was not possible to use those trials as baseline data for the statistical analysis. The upper curves are plotted to provide an estimate of the relative strength of the masking effect. The two lower curves represent the mean results for all three observers when the MS was presented. The two most effective masks of the 6-cycle/deg target were the 4- and 6-cycle/deg masking gratings. The 12-cycle/deg target was masked most strongly by the 12-cycle/deg mask.

A repeated-measures analysis of variance produced a significant main effect of the mask frequency $(\mathrm{p}<.01)$ and a significant TS by MS interaction $(\mathrm{p}<.01)$. The mask frequency effect may reflect the visual system's spatial frequency response, since the effective contrast of the higher and lower frequency masking gratings would be relatively attenuated, as indicated by measurements of the spatial modulation transfer function (Schober \& Hilz, 1965). The interaction effect indicates that the MS main effect depended on the TS frequency. In other words, which mask was most effective depended on which target was presented. As expected, the TS main effect was not significant $(p>.25)$, since each TS was compared to a $C S$ of the same frequency.

\section{DISCUSSION}

Some theories of metacontrast have attributed visual masking to lateral interaction between units 
that are sensitive to particular contours (Bridgeman, 1971; Weisstein, 1968). Thus, the closer the match between the contours of the mask and test, the greater the masking should be. In the present study, all masks have contours parallel to the contours of the test. but not all masks produce the same degree of masking. Since contours seem to be important, one might think that masking should increase as the number of contours is increased. In this study, however, the mask with the most contours, 24 cycles/deg, produced little masking. The spatial property of the stimuli that appears to be related to masking is the spatial frequency, not the proximity of contours or the number of contours.

Masking was greatest when the fundamental frequencies of the TS and MS were approximately the same, but matching of the fundamental frequencies is only a first approximation. The matching between TS and MS involves all the relevant spatial frequencies, as depicted in Figure 3. The spectra of the stimuli were computed by one-dimensional fast Fourier transforms of the luminance distributions across the horizontal midline of each stimulus. The effect of increasing the MS frequency is to displace the peak frequency to a higher value and spread apart the odd-numbered harmonic components.

We have simplitied the analysis in three ways: (1) The phase of the various frequency components is ignored: only the amplitude. the square root of the sum of the squares of the sine and cosine coefficients. is given at each frequency. (2) Only the horizontal midline analysis is presented, because most of the energy in the two-dimensional Fourier spectra represents the horizontal modulation of the stimulus gratings. (3) The energy spectra are not weighted by the spatial frequency sensitivity of the human visual system. although that would make comparisons between spectra more accurate. Since visual modulation transfer functions depend on the duration and wavelength of the stimulus, the choice of an appropriate weighting function would have involved some arbitrary assumptions.

If the TS spectra are compared with the MS spectra in Figure 3, then the correspondence in the frequency domain between the TS gratings and various MS gratings may be seen. For example, the 6 -cycle/deg MS has energy peaks centered at 6 and 18 cycles/deg, as does the 6-cycle/deg TS. The masking spectra differ from the target spectra in two respects: (1) Masking spectra have peaks at $1 \mathrm{cycle} / \mathrm{deg}$, due to the 1-deg gaps in the gratings. (2) Because of the scaling property of the Fourier transform, the target spectra have broader peaks than the masking spectra.

Slightly more masking of the 6-cycle/deg TS occurred with the 4-cycle/deg MS than with the 6-cycle/deg MS. The difference was small, and the two means did not differ significantly by Tukey's method of paired comparisons, but it was consistent

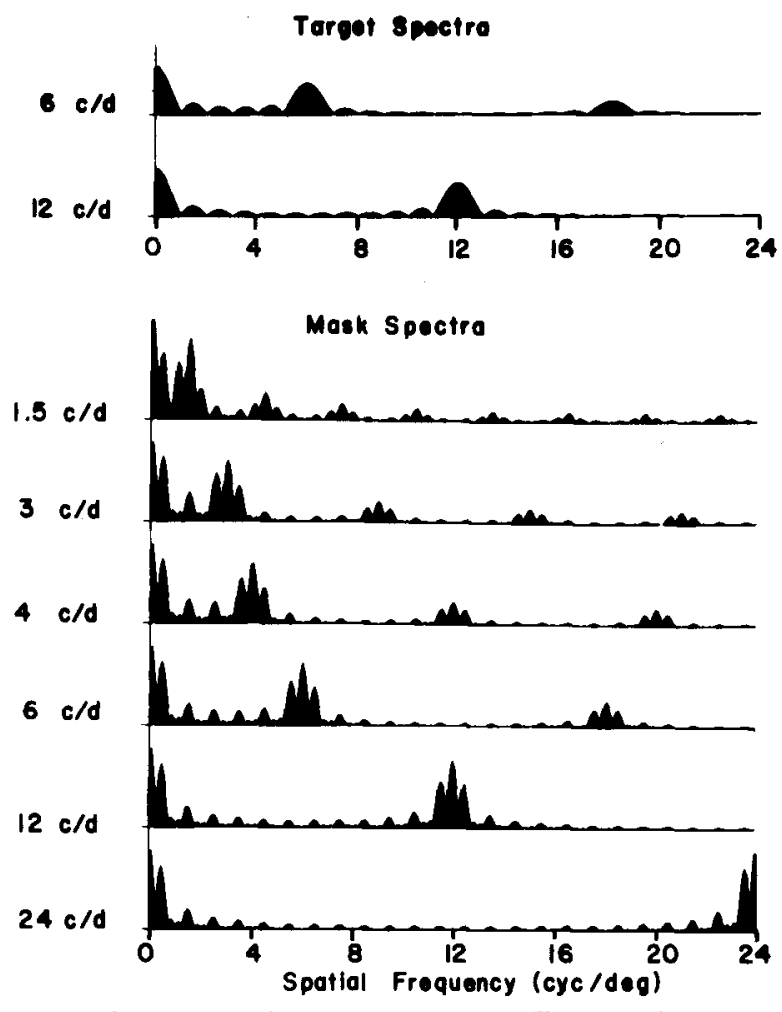

Figure 3. Spatial frequency spectra. The one-dimensional amplitude spectra were plotted from Fourier transforms of the luminance distributions across the horizontal midline of the masking stimuli (lower six curves) and the tanget stimuli (upper pair of curves).

for all three observers. If only the fundamental frequencies were considered, that fact might appear paradoxical. When the overall frequency spectra are considered, however, the spectrum of the 4-cycle/deg MS has considerable amplitude within 1 octave of the TS energy peaks, which might partially account for the effectiveness of the 4-cycle/deg MS.

One way to interpret the results shown in Figure 2 in terms of the general spatial frequency analysis approach is to suppose that some channels or units are sensitive to certain bands of spatial frequency and that such units have reciprocal inhibitory connections with other units which respond to the same spatial frequencies and which have nearby receptive fields. If the amount of inhibition were directly related to the excitatory level of the inhibiting unit. then a mismatch between its spatial sensitivity and the spatial frequencies of the stimulus would produce weak inhibition. thus little masking. For example, with a 6-cycle/deg TS and a MS of approximately the same frequency, the units whose receptive fields are near the TS would be highly stimulated, would produce a large amount of inhibition, and would result in considerable masking. Since other mask frequencies would stimulate the 6-cycle/deg flanking units less. they should produce lers inhibition and less masking of the TS. 
Masking gratings may change the appearance of other gratings in several ways. Although the observers described the masking effects as primarily reducing the clarity and apparent contrast of the targets, other features of the targets might be altered by the masking stimulus also. For example, the present interpretation suggests changes in the perceived spatial frequency of gratings as a consequence of metacontrast. A masking grating would be expected to reduce the visual system's sensitivity to the frequencies in the spectrum of the masking gratings. When a target stimulus is also presented, that part of its frequency spectrum corresponding to the mask's spectrum should be less well represented, and the changes in the target's effective spectrum would correspond to shifts in the target's apparent frequency.

There is some evidence to support the expectation of changes in perceived frequency. Blakemore and Sutton (1969) and Blakemore and Nachmias (1971) showed that adaptation to a grating produces shifts in apparent spatial frequency. Also, Klein, Stromeyer, and Ganz (1974) reported pronounced shifts in the apparent spatial frequency of gratings surrounded by other gratings of higher or lower frequency. Frome, Danielson, and Levinson (Note 2) extended the idea to two spatial dimensions and showed that adaptation to line gratings can affect the subsequent perception of the shape of rectangles. The studies just described were discussed in terms of long-term adaptation effects, but similar apparent frequency and shape effects may have much shorter time courses. It appears that metacontrast may provide the means of differentiating between explanations based on adaptation or fatigue, on the one hand, and those-based on transient neural inhibition, on the other.

\section{REFERENCE NOTES}

1. Armstrong, R., \& Sekuler, R. Spatial spread of orientationspecific masking. Paper presented at the Psychonomic Society meeting, 1972.

2. Frome, F., Danielson, J. T., \& Levinson, J. Z. Shifts in perception of size after adaptation to gratings. Paper presented at the meeting of the Association for Research in Vision and Ophthalmology, Sarasota, Florida, Spring 1974.

\section{REFERENCES}

Blakemore, C.. \& Nachmias, J. Orientation specificity of two visual aftereffects. Journal of Physiology, London, 1971, 213, 157-174.

Blakemore, C., \& Sutron. P. Size adaptation: A new aftereffect. Science, 1969, 166, 245-247.

Bridgeman. B. Metacontrast and lateral inhibition. Psychological Review. 1971, 78, 528-539.

Camprell, F. W. The transmission of spatial information through the visual system. In F. O. Schmitt \& F. G. Worden (Eds.). The neurosciences: Third study program. Cambridge, Mass: M.I.T. Press, 1974.

Campbell, F. W.. Cooper, G. F.. Robson, J. G., \& Sachs, M. B. Spatial selectivity of visual cells of the cat and the squirrel monkey. Journal of Physiology, London, 1969, 204, 120-121.

Campbell. F. W., \& Robson. J. G. Application of Fourier analysis to the visibility of gratings. Journal of Physiology. London. 1968, 197, 551-566.

Graham. N.. \& Nachmias. J. Detection of grating patterns containing two spatial frequencies: A comparison of singlechannel and multiple-channel models. Vision Research. 1971, 11, 251-259.

HaRmon, L. D.. \& Julesz, B. Masking in visual recognition: Effects of two-dimensional filtered noise. Science, 1973, 180. 1194-1197.

Klein, S.. Stromeyer, C. F., \& Ganz, L. The simultaneous spatial frequency shift: A dissociation between the detection and perception of gratings. Vision Research, 1974, 14. 1421-1432.

Lefton, L. A. Metacontrast: A review. Perception \& Psychophysics, 1973, 13(1B), 161-171.

Lovegrove. W. J., \& Over, R. Color adaptation of spatial frequency detectors in the human visual system. Science, 1972, 176. 541-543.

Maffei. L.. \& Fiorentini. A. Visual cortex as a spatial frequency analyzer. Vision Research, 1973, 13. 1255-1267.

Mayhew. J. E. W., \& Anstis, S. M. Movement aftereffects contingent on color. intensity, and pattern. Perception \& Psychophysics, 1972, 12.77-85.

Pollen, D. A., \& TaYlor, J. H. The striate cortex and the spatial analysis of visual space. In F. O. Schmitt \& F. G. Worden (Eds.). The neurosciences: Third study program. Cambridge, Mass: M.I.T. Press, 1974.

SChober. H. A. W., \& Hilz, R. Contrast sensitivity of the human eye for square-wave gratings. Journal of the Optical Society of America, 1965, 55, 1086-1091.

STROMEYER, C. F. Edge-contingent color aftereffects: Spatial frequency specificity. Vision Research, 1972, 12, 717-733.

STROMEYeR. C. F., \& Julesz, B. Spatial-frequency masking in vision: Critical bands and spread of masking. Journal of the Optical Society of America. 1972. 62. 1221-1232.

Weisstein. N. A Rashevsky-Landahl neural net: Simulation of metacontrast. Psychological Review, 1968, 75, 494-521.

Weisstein. N.. \& Bisaha. j. Gratings mask bars and bars mask gratings: Visual frequency response to aperiodic stimuli. Science. 1972, 176, 1047-1049.

(Received for publication September 10, 1975; revision accepted November $23,1975$. ) 